\title{
Parallel Programming Techniques \\ for Linear Algebra
}

\author{
L.D.J.C. Loyens
}

Koninklijke/Shell-Laboratorium, Amsterdam (Shell Research B.V.)

Postbus 3003, 1003 AA Amsterdam, The Netherlands.

\begin{abstract}
A number of parallel programming techniques have been used in the construction of parallel linear algebra algorithms at KSLA. These techniques rely on the use of invariants and clustering of invariants in order to obtain a parallel program consisting of $p$ processes with even work load distribution and low communication overhead. Absence of deadlock in communication processes is proved by using program text transformations.
\end{abstract}

\section{Introduction}

The theory proposed by Dijkstra [0] has been very fruitful in the design of sequential algorithms because it provides a rigorous base of understanding. In this theory programs and their corresponding correctness proofs are designed hand in hand. Some efforts have been made to extend the proof theory for sequential programming to the parallel case. The Gries-Owicki theory [5] is a good example of this. What is not dealt with in this theory is the development of parallel programs. The theory of designing parallel algorithms is still immature but a number of parallel programming techniques support the task of designing parallel algorithms. This paper tries to give an overview of the parallel programming techniques that have been used in the construction of a parallel linear algebra library at KSLA [9]. In this library many parallel algorithms have been designed, e.g. $L U$ - and $Q R$-decomposition, triangular system solving and matrix multiplication.

The programming techniques we discuss focuss on three important aspects during the design: functional specifications, invariants and correctness. The parallel program is specified using functional specifications in which an abstraction has been made of the communication behaviour of the program. This functional specification is the starting point for the construction of invariants. With every invariant a process is associated which will 
maintain it. Processes are clustered into a single parameterised process in order to obtain a parallel program consisting of $p$ processes with a low communication overhead and an even work load distribution. This parameterised process depends on the total number of processes $p$ and the chosen clustering. The necessary communication processes are derived from the invariant of such a clustered process. Advantages of this approach are that the parallel (linear algebra) program is derived independent of a given computer architecture and that a good insight can be obtained in the work load distribution and communication overhead. Actual implementations in a parallel programming language have to focuss on the implementation of the communication processes. In the implementation of the communication processes deadlock has to be avoided. Absence of deadlock in programs based on CSP [2] can be proved by transformation of the program texts. This technique is called PAR-simplification and is explained in a informal way in this paper.

The organisation of the paper is as follows. Sections 1 and 2 discuss functional specifications and (parallel) invariants. Section 3 looks at the use of auxiliary variables needed in both the derivation of a parallel program and in its correctness proof. The construction of an example linear algebra program including the clustering of processes and its impact on the total number of communications and the work load distribution is discussed in Section 4. Total correctness, especially absence of deadlock, is discussed in Section 5. Finally, conclusions and discussion points are given in Section 6.

The notation we use in this paper closely follows [1] and [0].

\section{Functional specifications}

The functional specification describes what a program must do. It is the abstraction of the application. Specifications of programs can be given in terms of pre- and postconditions [0]. A precondition is an assertion describing the set of initial states that must be satisfied to guarantee that a program, if terminated properly, reaches one of the states described by the postcondition.

Example 0 Consider the following functional specification of a program $S$

$$
\{Q: B: m \times n\} S\left\{R: b b t=B * B^{t}\right\} .
$$


The precondition $Q$ of program $S$ states that $B$ is an $m$ by $n$ matrix, and the postcondition $R$ of $S$ states that bbt is equal to $B$ times the transpose of $B$.

Functional specifications using pre- and postconditions are well-known in sequential programs. We also use them in parallel programs. In the linear algebra library, a specified program $S$ is always the parallel composition of $p$ parameterised processes. These processes can have a certain communication behaviour, but this will be specified in the functional specification of the communication processes. In this way, the communication behaviour remains hidden from the functional specification of the program $S$. Hence at this level, there is no functional distinction between a sequential and a parallel program.

\section{Parallel invariants}

An invariant of a program $S$ is an assertion expressing a relationship between variables that is invariant under execution of $S$. The use of invariants is well understood in sequential programming.

Example 1 Consider the following functional specification of program $S$

$$
\{Q: N \geq 0\}
$$

$S$

$$
\{R:(\mathbf{A} i: 0 \leq i<N: c[i]=A[i]+B[i])\}
$$

A sequential invariant for $S$ is $P$ seq, given by

$$
\text { Pseq }:(\mathbf{A} i: 0 \leq i<n: c[i]=A[i]+B[i]) \wedge 0 \leq n \leq N \text {. }
$$

The invariant of the last example clearly expresses that the summing of the elements of $A$ and $B$ should be done in some order. A parallel invariant does not contain such a sequential constraint. In such an invariant the freedom of expressing the order of operations is maintained, in such a way that this freedom can be used to exploit parallelism. Often non-determinism is present in a parallel invariant.

Example 2 A parallel invariant of example 1 is Ppar, given by

$$
\text { Ppar: }(\mathbf{A} i: i \in K: c[i]=A[i]+B[i]) \wedge K \subset\{0,1, \ldots, N-1\} \text {. }
$$




\section{The use of auxiliary variables}

A parallel invariant can be split up into local invariants. Each local invariant is the generalisation of a local postcondition belonging to one process. The local invariants often express relationships between local variables. In order to show correctness of parallel programs auxiliary variables are needed. An auxiliary variable is a variable not contained in the process text. Auxiliary variables relate local processes to each other.

Example 3 The local postconditions of example 2 are $R[i]: c[i]=A[i]+B[i], 0 \leq i<N$. The auxiliary variable is $K, K$ can be used to prove $|K|=N \wedge P$ par $\Rightarrow R$.

Local variables of processes can occur in proofs. In a process we do not use assertions expressed in terms of local variables of other processes. The reason for this is that by relating local variables to auxiliary variables non-interference proofs are avoided, which facilitates the use of the Gries-Owicki theory.

Example 4 Given are $N$ specifications of $N$ processes $S i, N \geq 1 \wedge 0 \leq i<N$,

$$
\begin{aligned}
& \{Q i: m i=M i\} \\
& \quad S i \\
& \left\{R i: m i=\left(\sum j: 0 \leq j<N: M j\right)\right\} .
\end{aligned}
$$

The variables $M i$ are auxiliary variables.

\section{The derivation of a parallel program}

After a brief discussion of the concepts of functional specifications, parallel invariants and auxiliary variables, we focus on the construction of a parallel program using these concepts. The example program is a variant of matrix multiplication. A similar derivation can also be given for programs like $L U$ - and $Q R$-decomposition.

A parallel program is constructed by starting with the functional specification of the problem. The postcondition is used to derive a parallel invariant, local postconditions and local invariants. Clustering of local invariants and postconditions can give a priori 
estimates of the maximum number of communications to be expected. This analysis motivates the kind of clustering thus defining the overall process structure. The construction of the program continues with the specification of the communication requirements. The communication processes are specified and designed separately.

The parallel program to be constructed is specified in example 0 . For the sake of simplicity we assume that $m$ is square, $m=q^{2}$. In order to obtain an invariant the postcondition $R$ is reformulated as

$$
R: \quad b b t=B * B^{t}
$$

$$
\equiv
$$$$
\left(\mathbf{A} u, v: 0 \leq u, v<m: b b t[u, v]=\left(\sum l: 0 \leq l<n: B[u, l] * B[v, l]\right)\right) .
$$

In the postcondition $R, m^{2}$ sums have to be calculated. We introduce for every calculation of a sum a process $(u, v)$, called an elementary process. Elementary process $(u, v)$ has as local variable $B[u, v]$. The local postcondition of one elementary process is

$$
R[u, v]: b b t[u, v]=\left(\sum l: 0 \leq l<n: B[u, l] * B[v, l]\right) .
$$

An invariant for process $(u, v)$ is derived by replacing the constant $n$ by a variable $k$. This results in a loop with as guard $k \neq n$. Note that $k$ is a local variable of process $(u, v)$, $k=k(u, v)$. The local invariant of one elementary process is

$$
P[u, v]: b b t[u, v]=\left(\sum l: 0 \leq l<k: B[u, l] * B[v, l]\right) \wedge 0 \leq k \leq n .
$$

The parallel invariant $P$ par is the conjunction of all the local invariants $P[u, v]$. Four clusterings of the elementary processes and its corresponding postconditions and invariants are considered : identity, row, block and grid. Every clustering, except the identity clustering, yields $q^{2}$ new processes. In order to calculate the value of $B * B^{t}[u, v]$ a number of elements of $B$ have to be communicated. Given a clustering, the total number of communications needed to calculate all values of $B * B^{t}[u, v]$ can be counted.

$$
\begin{aligned}
N[s, t](C)= & \text { the number of local postconditions containing } \\
& \text { element } B[s, t] \text { given clustering } C . \\
N \operatorname{com}(C)= & \left(\sum s, t: 0 \leq s<m \wedge 0 \leq t<n: N[s, t](C)-1\right) .
\end{aligned}
$$


$N \operatorname{com}(C)$ gives the total number of communications between the clusters in clustering $C$, because $B[s, t]$ needs only to be communicated to $N[s, t](C)-1$ clusters in a convenient distribution of the matrix elements. The four clusterings are :

$$
\begin{aligned}
\text { Ridentity }[u, v]= & R[u, v] \text { for } 0 \leq u, v<m, \\
\text { Rrow }[r i] \quad & (\mathbf{A} v: 0 \leq v<m: R[r i, v]) \text { for } 0 \leq r i<m, \\
R b l o c k[b i, b j]= & (\mathbf{A} u, v: 0 \leq u, v<m \wedge u \operatorname{div} q=b i \wedge v \operatorname{div} q=b j: R[u, v]) \\
& \quad \text { for } 0 \leq b i, b j<q, \\
\operatorname{Rgrid}[g i, g j]= & (\mathbf{A} u, v: 0 \leq u, v<m \wedge u \bmod q=g i \wedge v \bmod q=g j: R[u, v]) \\
& \quad \text { for } 0 \leq g i, g j<q .
\end{aligned}
$$

By counting $N[s, t]$ for these clusters the following results are obtained :

$$
\begin{array}{ll}
N[s, t](\text { identity })=2 * m-1, & N \operatorname{com}(\text { identity })=2 * m^{2} * n+\mathrm{O}(m * n), \\
N[s, t](\text { row }) & =m, \quad
\end{array}
$$

It is clear that a row clustering results in more communications than a grid or a block clustering. Hence, the choice to be made is between the latter two. It turns out that there is no difference between the grid and block clustering in the work load distribution and the number of communications per iteration. The grid clustering, and its corresponding distribution of matrices (cyclic storage) [4], is the key to achieving high efficiency in many parallel linear algebra computations, such as e.g. $L U$ - and $Q R$-decomposition and triangular system solving. Therefore, we also use assume a grid clustering in this example and we continue with the specification and derivation of the communication processes. For every cluster $(g i, g j)$ the following invariant Pgrid $[g i, g j]$ will be maintained

$$
\begin{gathered}
\operatorname{Pgrid}[g i, g j]:(\mathbf{A} u, v: 0 \leq u, v<m \wedge u \bmod q=g i \wedge v \bmod q=g j: \\
\left.b b t[u, v]=\left(\sum l: 0 \leq l<k: B[u, l] * B[v, l]\right)\right) \wedge \\
0 \leq k \leq n .
\end{gathered}
$$

We consider the effect of Pgrid $[g i, g j](k:=k+1)$ : 
$(\mathbf{A} u, v: 0 \leq u, v<m \wedge u \bmod q=g i \wedge v \bmod q=g j:$

$$
\begin{aligned}
& \left.b b t[u, v]=\left(\sum l: 0 \leq l<k: B[u, l] * B[v, l]\right)+B[u, k] * B[v, k]\right) \wedge \\
& 0 \leq k+1 \leq n
\end{aligned}
$$

The latter conjunct is implied by Pgrid[gi,gj] $\wedge k \neq n$, and for the first conjunct, the values a cluster $(g i, g j)$ needs have to be communicated. Therefore we introduce assertions Pcomrow $[g i, g j]$ and Pcomcol $[g i, g j]$ given by

$$
\begin{aligned}
& \text { Pcomrow }[g i, g j]:(\text { A } u: 0 \leq u<m \wedge u \bmod q=g i: x[u]=B[u, k]), \\
& \text { Pcomcollgi,gj]: (A } v: 0 \leq v<m \wedge v \bmod q=g j: y[v]=B[v, k]) .
\end{aligned}
$$

The communication obligations are imposed by Pcomrow $[g i, g j]$ and Pcomcol $[g i, g j]$. It is supposed that the matrix $B$ is divided among $q^{2}$ processes using a grid distribution. This means that cluster $(g i, g j)$ owns the matrix elements given by set $O[g i, g j]$,

$$
O[g i, g j]=\{u, v: 0 \leq u, v<m \wedge u \bmod q=g i \wedge v \bmod q=g j:((u, v), B[u, v])\}
$$

Notice that for a fixed $g$ i all clusters $(g i, g j)$, with $0 \leq g j<q$ and the same value of $k$, need the set $N(g i)$ given by :

$$
N(g i)=\{u: 0 \leq u<m \wedge u \bmod q=g i:((u, k), B[u, k])\}
$$

The communication process needed to establish Pcomrow $[g i, g j]$ can be specified by

$$
\begin{aligned}
& \{\text { Qcomrow }[g i, g j]: g j \neq g x \vee N[g i] \subset O[g i, g j]\} \\
& \text { Comrow }[g i, g j] \\
& \{\text { Pcomrow }[g i, g j]:\{u: 0 \leq u<m \wedge u \bmod q=g i:((u, k), x[u])\}=N(g i)\},
\end{aligned}
$$

with $g x$ satisfying $q x=k \bmod q$.

Per iteration, $|N(g i)|$ values will be communicated from cluster $(g i, g x)$ to all clusters $(g i, g j), 0 \leq g j<q, g j \neq g x$. Such a broadcast can efficiently be implemented using pipelining on a number of computer architectures, like a mesh, a torus, or a hypercube. The time complexity of the communication process Comrow $[g i, g j]$ is $\mathrm{O}(|N(g i)|$ $+q)=\mathrm{O}(m / q+q)$ A specification of the communication process needed to establish Pcomcol $[g i, g j]$ is almost similar and is not given. 
The time complexity of the cluster $(g i, g j)$, and also of the parallel program, is the time complexity of the communication processes plus the time complexity to update $b b t[u, v]$ by $B[u, k] * B[v, k]=x[u] * y[v]$. The time complexity of the communication processes is $\mathrm{O}(n *(|N(g i)|+|N(g j)|+2 * q))=\mathrm{O}(n *(m / q+q))=\mathrm{O}(n * \sqrt{m})$. The time complexity to do the update is $\mathrm{O}(n *|N(g i)| *|N(g j)|)=\mathrm{O}\left(n *\left(m^{2} / q^{2}\right)\right)=\mathrm{O}(n * m)$. Hence, the resulting time complexity for $m=q^{2}$ processes is $\mathrm{O}(n * m)$. The amount of work to be done by every increase of $k$ is the same; $k$ can be seen as a distributed global clock. This implies that the load is well balanced among the clusters $(g i, g j)$.

In general there are $p=Q^{2}$ processes, $1 \leq Q \leq q$. If this is the case then the work load distribution is not the same for every proces because the number of elements in the sets $N(g i)$ satisfies $m \operatorname{div} Q \leq|N(g i)| \leq m$ div $Q+1$. A load imbalance can occur by doing the update with differently sized sets. If this is the case then the maximal time difference is of $\mathrm{O}(2 * m$ div $Q+1)$, but this term is of lower order than the time complexity of the program which is $\mathrm{O}\left(n * m^{2} / Q^{2}\right)+\mathrm{O}(n *(m / Q+Q))$. Hence, for $p=Q^{2}$ processes, an efficient program with relatively even work load distribution and a low communication overhead can be obtained.

\section{Total correctness}

Partial correctness of programs only considers if the program is correct with respect to its specification. A total correctness proof gives a proof of termination (if desired), absence of deadlock and individual starvation. Termination of a sequential program is shown by defining a variant function in the usual way. Termination of a parallel program can be shown by proving that every sequential process terminates and also that the parallel execution of processes does not invalidate the proof of sequential termination of the processes [5]. Absence of deadlock can be shown in many ways, for example by defining partial orders [2]. Absence of deadlock in the parallel linear algebra library [9] is shown by applying PAR simplification. The communication model that is used is based on CSP [2] and occam [3]. Processes communicate synchronously with each other using point-to-point channels and two communication primitives. The primitives are $c !$ and $c$ ?

PAR-simplification makes use of the occam programming model [3]. Processes in 
occam are only blocked at communication primitives (abortion of the IF statement and the occurence of timers are neglected). This observation suggests a method of proving absence of deadlock in occam programs. Take a parallel program and try to simplify the program text in such a way that presence or absence of deadlock is preserved. This is called deadlock invariance. An occam program can be simplified using the following base rule :

PAR

c!

c? simplifies to SKIP.

In general PAR-simplification is based on two invariances : deadlock invariance and communication invariance. Communication invariance means that a subprocess of a process can be substituted by another subprocess which has the same external communication behaviour. In order to simplify parallel programs based on CSP, mathematical induction and the introduction of auxiliary variables is often necessary. A formal model of PARsimplification cannot be given in this paper. A large body of other transformation rules is contained in [7]. The next example will clarify the application of PAR-simplification.

Example 5 Consider the following occam program text $\mathrm{S}(\mathrm{n}), \mathrm{c}$ is an array of channels,

$\mathrm{S}(\mathrm{n}):$

PAR $\mathrm{i}=[0$ FOR $\mathrm{n}]$

SEQ

c[i]!

$c[i+1]$ ? simplifies to

$T(N):$

SEQ

c[0]!

$\mathrm{c}[\mathrm{n}] ?$ 
A proof of the last example can be given using mathematical induction on the program text.

\section{Proof of example 5}

(i) $n=1$, trivial.

(ii) suppose $\mathrm{S}(\mathrm{n}), n \geq 1$, simplifies to $\mathrm{T}(\mathrm{n})$, $\mathrm{S}(\mathrm{n}+1)$ :

PAR $i=[0$ FOR $n+1]$

SEQ

c[i]!

$c[i+1]$ ? simplifies to $\{$ range splitting $\}$

PAR

$\operatorname{PAR} \mathrm{i}=[0 \mathrm{FOR} \mathrm{n}]$

SEQ

c[i]!

$\mathrm{c}[\mathrm{i}+1]$ ?

SEQ

$\mathrm{c}[\mathrm{n}] !$

$c[n+1]$ ? simplifies to $\{$ induction hypothesis, communication invariance $\}$

PAR

SEQ

c[0]!

$\mathrm{c}[\mathrm{n}]$ ?

SEQ

c[n]!

$c[n+1]$ ? simplifies to $\{$ variance of base rule $\}$

SEQ

c[0]!

$\mathrm{c}[\mathrm{n}+1]$ ? . 


\section{Conclusions and discussion points}

The parallel programming techniques that have been used in the construction of the linear algebra library are based on functional specifications and parallel invariants. In the development of a parallel program performing $B * B^{t}$ a number of steps are encountered. The clustering of local postconditions and invariants gives insight into the maximum number of communications. An optimally load balanced parallel program for $B * B^{t}$ using $m=q^{2}$ processes and a grid distribution of the matrix can in this way be derived with time complexity $\mathrm{O}(m * n)$ and a maximal number of communications of $\mathrm{O}\left(m^{3 / 2} * n\right)$. The generalisation for $p=Q^{2}$ processes, $1 \leq Q \leq q$, is straightforward and results in a parallel program with time complexity $\mathrm{O}\left(n * m^{2} / p\right)+\mathrm{O}(n * m / \sqrt{p})$. The derivation of the program is given indepent from a given computer architecture.

The application of PAR-simplification, touched upon briefly in this paper, seems to be a good alternative to prove absence or presence of deadlock in parallel programs. The full scope of this technique is still not clear. Some applications of PAR-simplification in the linear algebra library showed that it is possible to deal with asynchronous communication, provided that messages eventually arrive in the same order as they were sent, and it is also possible to deal with communication processes that have a dynamic communication structure. This claim is only for processes with a fixed number of channels. Another discussion point concerns the use of functional languages as specifications for parallel programs. Functional languages may provide good alternatives to derive recursive parallel programs, i.e. parallel programs using dynamic process creation. An example of the connection between functional languages and parallel programs in this context can be found in [6]. The derivation of the example parallel program performing $B * B^{t}$ started in a 'classical' way leading to what can be called a 'cell' invariant in systolic computations [8]. By introducing clustering the granularity of the computation can be controlled, thus making it possible to minimise the number of communications and to assure load balance. It would be interesting to look how clustering would affect systolic computations. The program derived in $[8]$ can be treated in the same way as the example parallel program in this paper. 


\section{References}

[0] E.W.D. Dijkstra, "A Discipline of Programming," Prentice-Hall, 1976.

[1] D. Gries, "The Science of Programming," Springer-Verlag New York Inc., 1981.

[2] C.A.R. Hoare, "Communicating Sequential Processes," Prentice-Hall International, UK, Ltd., London, 1985.

[3] Inmos Ltd., "The occam programming manual," Prentice-Hall, 1984.

[4] S.L. Johnsson, "Communication Efficient Basic Linear Algebra Computations on Hypercube Architectures," J. Sci. Statist. Comput. 4 (1987).

[5] S. Owicki, D. Gries, "An axiomatic proof technique for parallel programs I," Acta Informatica 6 (1976).

[6] M. Rem, "Small Programming Exercises 18, 19 and 20," Science of Computer Programming 7,8 and 9 (1986).

[7] A.W. Roscoe, C.A.R. Hoare, "The laws of occam programming," PRG-53, Oxford University Computing Laboratory.

[8] J.L.A. van de Snepscheut, "A derivation of a distributed implementation of Warshall's algorithm," Science of Computer Programming 7 (1986).

[9] J.G.G. van de Vorst, "The internal structure of a parallel Linear Algebra library," Submitted to Distributed Computing. 